\title{
The next step in improving (semi-)quantitative MPI PET
}

\author{
S. S. Koenders, MSc (1), ${ }^{\mathrm{a}, \mathrm{c}}$ J. A. van Dalen, PhD, ${ }^{\mathrm{b}}$ and J. D. van Dijk, MSc, PhD $\mathbb{0}^{\mathrm{a}}$ \\ a Department of Nuclear Medicine, Isala Hospital, Zwolle, The Netherlands \\ b Department of Medical Physics, Isala Hospital, Zwolle, The Netherlands \\ c Technical Medical Center, University of Twente, Enschede, The Netherlands
}

Received Aug 14, 2020; accepted Aug 14, 2020

doi: $10.1007 / \mathrm{s} 12350-020-02346-3$

\section{See related article, pp. 449-460}

\section{BACKGROUND}

Myocardial blood flow (MBF) quantification with PET results in an improved risk assessment of coronary artery disease (CAD) in addition to semi-quantitative myocardial perfusion imaging. ${ }^{1} \mathrm{~A}$ decrease in the myocardial flow reserve (MFR, MBF in stress/MBF in rest) or an increasing area of perfusion deficits results in a higher risk on cardiac mortality, as shown in Figure 1. To be able to quantify MBF, tracer uptake in the left ventricle and the myocardium during the scan has to be calculated from the imaging data. However, in the process of data acquisition, reconstruction, post-processing and interpretation of the data, there are several pitfalls that one should be aware of as these pitfalls can result in unreliable uptake calculations and hence MBF measurements. ${ }^{2}$

Multiple pitfalls for MBF quantification and corresponding solutions have been identified in the last decade. First, it is important that the PET scanner has a sufficient count-rate capability to prevent detector saturation during the first-pass phase which can lead to artificially high MBF values. ${ }^{3,4}$ Secondly, to increase the reproducibility of MBF measurements, a constant activity infusion profile is required. ${ }^{5}$ Yet not all Strontium82-Rubidium-82 generators are able to produce such a constant activity bolus. Third, misregistration of PET

Reprint requests: J. D. van Dijk, MSc, PhD, Department of Nuclear Medicine, Isala Hospital, PO Box 104008000 GK Zwolle, The Netherlands; jorisvdijk@gmail.com

J Nucl Cardiol 2022;29:461-3.

$1071-3581 / \$ 34.00$

Copyright (c) 2020 American Society of Nuclear Cardiology. with CT data that is used for attenuation correction can result in altered MBF measurements. ${ }^{6}$ Fourth, reconstruction settings and post-processing software packages can result in a MBF bias as well. ${ }^{7,8}$ Although the reproducibility is often unaffected when changing reconstruction settings or shifting to different software, a bias may occur. Fifth, patient motion and repositioning of the heart after administration of a stress agent can also negatively affect the reliability of MBF measurements. ${ }^{9,10}$ However, this influence can be limited by correcting the dynamic time-frames for possible motion and "myocardial creep". ${ }^{11}$ Last, the test-retest reliability of MBF quantification is relatively large with a typical uncertainty of $21 \%{ }^{12}$ This implies that there is a large 'grey area' in distinguishing reduced and normal MBF values and physicians should be aware of this during interpretation.

Although our knowledge of and solutions to pitfalls increases, several issues remain. For example, how to prevent scans in which there is not a well-defined activity bolus? Or how to correct for a delayed myocardial activity uptake in patients with coronary artery bypass grafts which may hamper MBF quantification. Or how to deal with MBF measurements that are based on different administered activities?

In this issue of the Journal of Nuclear Cardiology, Hoff et al. ${ }^{13}$ addressed the latter issue by studying the effect of the injected Rubidium-82 activity on the MBF measurements and the relative myocardial perfusion images. They scanned forty patients twice using the lowest tracer activity of $1110 \mathrm{MBq}$ as recommended in the prescribing information versus either $740 \mathrm{MBq}$ of $370 \mathrm{MBq}$. Next, they compared the quantitative outcomes by comparing the MBF and MFR measurements and relative outcomes by comparing the ejection fraction (EF) and the semi-quantitative parameter total perfusion deficit (TPD). 


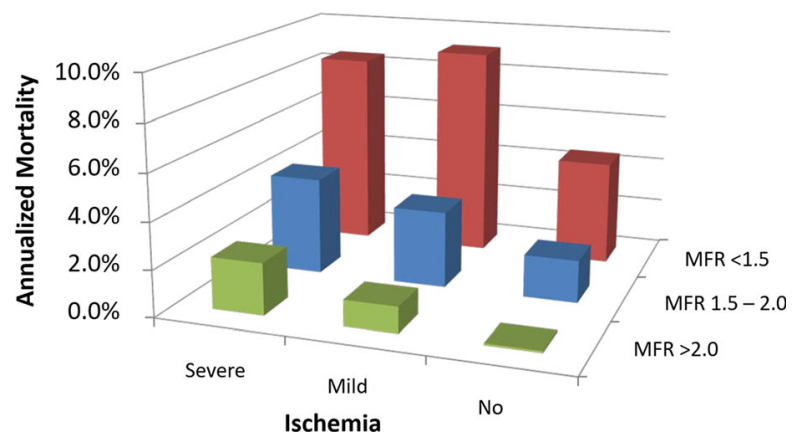

Figure 1. Annualized mortality by myocardial flow reserve (MFR) and severity of ischemia adapted from Murthy et al. ${ }^{1}$ based on PET MPI examinations of 2783 patients and a median follow-up of 1.4 years (IQR: 0.7-3.2 years). With increasing ischemia and decreasing MFR, the annualized mortality increases.

Hoff et al. reported that peak count rate per injected activity decreased and peak dead time correction factors increased due to detector saturation in the first-pass phase. Reducing the activity from $1100 \mathrm{MBq}$ to 740 and further to $370 \mathrm{MBq}$ led to a bias and decreasing correlations for TPD and EF. This can be explained by the decreasing count statistics in the late tissue phase when lowering the administered activity. Acceptable image quality for visual interpretation was still obtained when using $740 \mathrm{MBq}$ but the images became too noisy when using $370 \mathrm{MBq}$.

Although the authors showed that a lower tracer activity affects the image quality, MBF quantification was only marginally different. The global and regional MBF measurements correlated well for both activities (370 MBq and $740 \mathrm{MBq}$ ) with $1110 \mathrm{MBq}\left(R^{2}>0.97\right)$. Good correlations $\left(R^{2}=0.96\right)$ were also found for global MFR regarding both activities $(370 \mathrm{MBq}$ and $740 \mathrm{MBq}$ ) to $1110 \mathrm{MBq}$ and no bias was observed. An injected activity of $740 \mathrm{MBq}$ resulted in a small positive bias in global and regional (RCA and LCX) MBF values as compared to $1110 \mathrm{MBq}$. Using $370 \mathrm{MBq}$ resulted in a small negative MBF bias on a global level and specifically in the LAD territory, as compared to $1110 \mathrm{MBq}$. To summarize, it seems that the low tracer activity of $740 \mathrm{MBq}$ is sufficient for both semi-quantitative and quantitative MPI while the injected tracer activity of 370 $\mathrm{MBq}$ only provided acceptable quantitative MPI as compared to $1110 \mathrm{MBq}$.

Two comments can be made considering the results obtained by Hoff et al. First, they reported a lower mean peak dead time factor for conventional PET using photomultiplier tubes (PMT) than for new generation PET using solid-state photodetectors at a comparable activity. This suggests that the optimal activity regime for the new generation PET scanners is lower than for conventional PET scanners using PMT. This is remarkable as other studies show better count-rate capability for PET scanners based on solid-state photodetectors as compared to PMT PET. ${ }^{3,4}$ Secondly, it is questionable if lowering the activity to $740 \mathrm{MBq}$, as suggested by Hoff et al., can be generalized to previous generation PMT PET scanners as they often have low count-rate capabilities. ${ }^{3,4}$

Hoff et al. showed that both MBF and MFR measurements correlated well between the reduced administered $\mathrm{Rb}-82$ activity (740 MBq) and the recommended Rb-82 activity (1100 MBq). However, where MFR resulted in no bias but large limits of agreement, biases were observed for MBF with smaller limits of agreement. This raises an important question: should we use stress MBF or MFR, or perhaps both, in clinical practice? Literature still shows conflicting answers regarding this question. Some suggest that stress MBF is the preferred parameter ${ }^{14}$ while others propose MFR as the parameter to use. ${ }^{15,16}$ In addition, Gould et al. ${ }^{17}$ suggest a method that integrates the stress MBF and MFR into a parameter called coronary flow capacity. However, the clinical value of this parameter over MFR or stress MBF has yet to be determined. Furthermore, MBF and MFR measurements are often only assessed on a global or vascular level whereas visual images are assessed on smaller regional levels. Hence, it is still unknown whether we should use MBF, MFR or coronary flow capacity and whether we should do this on a global, vascular or regional level to obtain the best prognostic information.

Altogether, the study of Hoff et al. clearly shows that the amount of tracer activity to be administered should be chosen with care for MBF and MFR measurements using PET. Therefore, each center that wants to start with MBF quantification should determine the activity that results in sufficient count statistics for relative perfusion imaging or semi-quantitative imaging but does not lead to detector saturation during the firstpass phase for MBF or MFR quantification. The study by Hoff et al. has extended our current knowledge on the (technical) pitfalls in MBF and MFR quantification using PET. This greatly contributes to integration of flow quantification in clinical practice. Solving the remaining issues will likely result in even more reliable blood flow measurements and thus a further improvement in the diagnostic accuracy and prognostic value of PET MPI in the near future. 


\section{References}

1. Murthy VL, Naya M, Foster CR, Hainer J, Gaber M, Di Carli G, et al. Improved cardiac risk assessment with noninvasive measures of coronary flow reserve. Circulation. 2011;124:2215-24.

2. van Dijk JD, Jager PL, van Dalen JA. Pitfalls in myocardial blood flow quantification with rubidium-82 PET. Tijdschr voor Nucl Geneeskd. 2017;39:1822-9.

3. Renaud JM, Yip K, Guimond J, Trottier M, Pibarot P, Turcotte E, et al. Characterization of 3-dimensional PET systems for accurate quantification of myocardial blood flow. $J$ Nucl Med. 2017;58:103-9.

4. van Dijk J, Jager P, van Osch J, Khodaverdi M, van Dalen J. Comparison of maximal rubidium-82 activities for myocardial blood flow quantification between digital and conventional PET systems. J Nucl Cardiol. 2019;26:1286-91.

5. Klein R, Ocneanu A, Renaud J, Ziadi M, Beanlands R, deKemp R. Consistent tracer administration profile improves test-retest repeatability of myocardial blood flow quantification with $82 \mathrm{Rb}$ dynamic PET imaging. J Nucl Cardiol. 2018;25:929-41.

6. Rajaram M, Tahari AK, Lee AH, Lodge MA, Tsui B, Nekolla S, et al. Cardiac PET/CT misregistration causes significant changes in estimated myocardial blood flow. J Nucl Med. 2013;54:50-4.

7. Tahari AK, Lee A, Rajaram M, Fukushima K, Lodge MA, Lee $\mathrm{BC}$, et al. Absolute myocardial flow quantification with (82)rb PET/CT: Comparison of different software packages and methods. Eur J Nucl Med Mol Imaging. 2014;41:126-35.

8. Armstrong I, Tonge C, Arumugam P. Impact of point spread function modeling and time-of-flight on myocardial blood flow and myocardial flow reserve measurements for rubidium-82 cardiac PET. J Nucl Cardiol. 2014;21:467-74.

9. Koenders SS, van Dijk JD, Jager PL, Ottervanger JP, Slump CH, van Dalen JA. Impact of regadenoson-induced myocardial creep on dynamic rubidium-82 PET myocardial blood flow quantification. J Nucl Cardiol. 2019;26:719-28.

10. Memmott M, Tonge C, Saint K, Arumugam P. Impact of pharmacological stress agent on patient motion during rubidium-82 myocardial perfusion PET/CT. J Nucl Cardiol. 2018;25:1286-95.
11. Koenders SS, van Dijk JD, Jager PL, Ottervanger JP, Slump CH, van Dalen JA. How to detect and correct myocardial creep in myocardial perfusion imaging using rubidium-82 PET? J Nucl Cardiol. 2019;26:729-34.

12. Kitkungvan D, Johnson NP, Roby AE, Patel MB, Kirkeeide R, Gould KL. Routine clinical quantitative rest stress myocardial perfusion for managing coronary artery disease: Clinical relevance of test-retest variability. JACC Cardiovasc Imaging. 2017;10:56577.

13. Hoff CM, Sørensen J, Christensen NL, Bouchelouche K, Tolbod L. Activity regimes for $82 \mathrm{Rb}$ cardiac PET: Effects on absolute MBF and MPI. J Nucl Cardiol. 2020. https://doi.org/10.1007/s12 350-020-02266-2 (Epub ahead of print).

14. Farhad H, Dunet V, Bachelard K, Allenbach G, Kaufmann PA, Prior JO. Added prognostic value of myocardial blood flow quantitation in rubidium-82 positron emission tomography imaging. Eur Heart J Cardiovasc Imaging. 2013;14:1203-10.

15. Ziadi MC, deKemp RA, Williams KA, Guo A, Chow BJ, Renaud $\mathrm{JM}$, et al. Impaired myocardial flow reserve on rubidium-82 positron emission tomography imaging predicts adverse outcomes in patients assessed for myocardial ischemia. Cardiac imaging. 2011;58:740-8.

16. Patel KK, Spertus JA, Chan PS, Sperry BW, Al Badarin F, Kennedy KF, et al. Myocardial blood flow reserve assessed by positron emission tomography myocardial perfusion imaging identifies patients with a survival benefit from early revascularization. Eur Heart J. 2020;41:759-68.

17. Gould KL, Johnson NP, Roby AE, Nguyen T, Kirkeeide R, Haynie $\mathrm{M}$, et al. Regional, artery-specific thresholds of quantitative myocardial perfusion by PET associated with reduced myocardial infarction and death after revascularization in stable coronary artery disease. J Nucl Med. 2019;60:410-7.

Publisher's Note Springer Nature remains neutral with regard to jurisdictional claims in published maps and institutional affiliations. 